\title{
Influence of the HLA-DQB1*0201 allele on the immune response in a Schistosoma mansoni infection
}

\author{
Eliana A.G. Reis ${ }^{a}$, Daniel A. Athanazio ${ }^{a}, b$, Alan J.A. McBride ${ }^{a}$, Theomira Mauadi Carmo Azevedo ${ }^{a}$, \\ Isis F. Magalhães-Santos ${ }^{a}$, Donald Harn ${ }^{c}$, Mitermayer G. Reis ${ }^{\mathrm{a}, \mathrm{b}, *}$ \\ a Oswaldo Cruz Foundation (FIOCRUZ), Salvador, Bahia, Brazil \\ ${ }^{\mathrm{b}}$ Federal University of Bahia, Bahia, Brazil \\ ${ }^{\mathrm{c}}$ Department of Immunology and Infectious Diseases, Harvard School of Public Health, Boston, MA, USA
}

\section{A R T I C L E I N F O}

\section{Article history:}

Available online 15 April 2008

\section{Keywords:}

HLA

Schistosomiasis

Schistosoma mansoni

Immunoglobulin isotype

\begin{abstract}
A B S T R A C T
We previously reported the association of the major histocompatibility complex class II HLA-DQB $1^{*} 0201$ allele with hepatosplenic schistosomiasis. The aim of this study was to evaluate the cytokine responses of peripheral blood mononuclear cells (PBMCs) and the serum levels of immunoglobulin isotypes. The study population was selected from a schistosomiasis endemic area. No significant differences in cytokine profiles were detected in PBMCs stimulated with Schistosoma mansoni soluble egg antigen (SEA), regardless of the subjects DQB1*0201 genotype or infection status. However, previously infected DQB $1^{*} 0201$ positive individuals had significantly lower levels of IgG4 compared to DQB1*0201 negative individuals $(P<0.05)$.

(C) 2008 Elsevier B.V. All rights reserved.
\end{abstract}

The major outcome of a Schistosoma mansoni infection is chronic hepatosplenic schistosomiasis in which granulomatous inflammation and fibrosis of the liver leads to potentially fatal portal hypertension (Andrade, 2004). Approximately 5\% of individuals living in S. mansoni endemic areas develop hepatosplenic disease despite treatment (José C. Bina, personal communication). The investigation of host genetic determinants for disease severity is a developing field in schistosomiasis research, and certain major histocompatibility complex (MHC) alleles have been associated with schistosomal hepatosplenomegaly. Among Egyptian individuals, positive associations were reported for human leukocyte antigen (HLA) A1 and B5 (Salam et al., 1979), A2 and B12 (Hafez et al., 1991), B5 and DR3 (Assaad-Khalil et al., 1993) and a gamma interferon (IFN- $\gamma$ ) gene polymorphism (Blanton et al., 2005). In a study in Southern Brazil, however, no association was observed between HLA-A1 or B5 expression and hepatomegalic disease (Cabello et al., 1991). In northeastern Brazil, we previously studied three loci (DRB1, DQA1, and DQB1) and detected an association between DQB1*0201 and hepatosplenic disease (Secor et al., 1996). The same allele, especially in combination with DRB ${ }^{*} 0301$, was subsequently associated with post-schistosomal hepatic fibrosis caused by Schistosoma

\footnotetext{
* Corresponding author at: Oswaldo Cruz Foundation-Ba, Centro de Pesquisas Gonçalo Moniz-CPqGM, Rua Waldemar Falcão, 121, Candeal, CEP: 40296-710, Salvador, Bahia, Brazil. Tel.: +55 71 31762200; fax: +55 7131762326 .

E-mail address: miter@bahia.fiocruz.br (M.G. Reis).
}

japoni (S. japonicum) infection in Chinese patients (Hirayama et al., 1999).

It is possible that these genetic determinants may interfere in schistosomal pathogenesis and the host immune response. For instance, late stage human infection is characterized by polarization from a potentially protective Th1-type immune response to a Th2type response (Pearce and MacDonald, 2002). In addition, there is strong evidence from animal models to support the involvement of a Th2-type response in the severity of granulomatous inflammation and fibrosis of the liver (Farah et al., 2000; Reiman et al., 2006). However, the association is unclear in humans as peripheral blood mononuclear cell (PBMC) responses have been associated with higher levels of either tumor necrosis factor alpha (TNF- $\alpha$ ) (Mwatha et al., 1998) or interleukin (IL)-13, IL-4, IL-5 and IL-10 that have been linked to progressive or end-stage liver disease (de Jesus et al., 2004; Montenegro et al., 1999; Alves Oliveira et al., 2006). An additional mechanism may involve the expression of different immunoglobulin isotypes as they have been associated with protection and susceptibility to re-infection (Demeure et al., 1993; Caldas et al., 2000). The present report focuses on the influence of the HLA-DQB1 ${ }^{*} 0201$ allele on immune response-related cytokine production and immunoglobulin isotype production.

The study population was selected from a schistosomiasis endemic area (Itaquara, Bahia, Brazil). Field studies have been carried out at this location since 1989 when the overall prevalence of S. mansoni infection was $90 \%$. Annual surveys are carried out in this region for schistosomiasis and other helminth infections and any infected individuals are subsequently treated. Thirty-three 
individuals resident in the endemic region and previously treated for a $S$. mansoni infection were selected for inclusion in this study. Upon re-evaluation, 23 volunteers were negative by stool sample for $S$. mansoni eggs (egg-negative), while 10 individuals had been reinfected (egg-positive) by S. mansoni. The HLA-DQB1*0201 status of the egg-negative (previously infected) group was determined as negative $(n=14)$ or positive $(n=9)$ as described previously (Secor et al., 1996). Egg-positive (re-infected) individuals included seven positive individuals and three negative individuals for the HLA-DQB $1{ }^{*} 0201$ genotype. All individuals provided informed written consent and samples were collected according to protocols approved by the Human Ethical Committee of the Oswaldo Cruz Foundation. All infected individuals in the study were subsequently treated for schistosomiasis and other parasitic infections.

Peripheral blood mononuclear cells (PBMCs) were isolated from $20 \mathrm{ml}$ heparinized blood by density gradient centrifugation on Ficoll Histopaque (Sigma-Aldrich, St. Louis, MO, USA) as described previously (Velupillai et al., 2000). PBMCs were cultured with soluble egg antigen (SEA) from S. mansoni $(5 \mu \mathrm{g} / \mathrm{ml})$, Concanavalin A $(5 \mu \mathrm{g} / \mathrm{ml})$, or medium alone as a control. SEA was prepared as described elsewhere (Harn et al., 1989). Supernatants were collected at $24 \mathrm{~h}$ for IL-10 and TNF- $\alpha$ and at $72 \mathrm{~h}$ for IL-5 and IFN- $\gamma$. Sandwich ELISAs were performed following the manufacturer's instructions for TNF- $\alpha$, Duo-set kit (R\&D System, Minneapolis, MN, USA); IFN- $\gamma$ kit from Genzyme (Cambridge, MA, USA) and IL-5 and IL-10 kits from PharMingen (BD, Mountain View, CA, USA).

Specific levels of $\operatorname{IgM}$, IgA, IgE, IgG1, IgG2, IgG3 and IgG4 against S. mansoni SEA were analyzed by ELISA, as described previously (Li et al., 1995). Briefly, microtiter plates were incubated with the following antibodies diluted in PBS-Tween 80 (0.05\%): monoclonal anti-human IgA (1:15,000), anti-human IgE (1:2500), purified antihuman IgM $(1: 15,000)$ conjugated to peroxidase and monoclonal anti-human IgG1 (1:2000), IgG2 (1:15,000), IgG3 (1:2000) and IgG4 $(1: 15,000)$ conjugated to biotin (Sigma-Aldrich). Tetramethylben- zidine $(\mathrm{TMB})$ and peroxidase substrate solution $\mathrm{B}(1: 1)$ were used for detection of peroxidase activity (Kirkegaard \& Perry Laboratories, Gaithersburg, MD, USA). The reaction was stopped with $2 \mathrm{~N}$ $\mathrm{H}_{2} \mathrm{SO}_{4}$ and read at $450 \mathrm{~nm}$ by a spectrophotometer linked to MDSSoftmax software. Cut-off values for the ELISA were calculated using sera collected from a group of individuals $(n=17)$ resident in the city of Salvador, a non-endemic region for schistosomiasis. The cut-off value was calculated as the mean optical density $\left(\mathrm{OD}_{450}\right)$ plus three standard deviations and was determined to be 0.14 . Statistical analyses were performed using non-parametric Mann-Whitney and Kruskal-Wallis tests using GraphPad Prism 4.0 software (GraphPad Software, San Diego, CA, EUA), $P$-values $<0.05$ were considered significant in this study.

No significant differences in cytokine production were detected following SEA stimulation of PBMCs from egg-positive or eggnegative individuals (Fig. 1) regardless of their DQB1*0201 status. In addition, no significant differences were observed after Concanavalin A stimulation.

In the present study, SEA stimulated PBMCs from previously infected DQB1*0201 positive individuals produced significantly lower levels of anti-SEA IgG4 compared to previously infected DQB1 ${ }^{*} 0201$ negative individuals $(P<0.05$, Fig. 2$)$. Among this group, $57.1 \%(8 / 14)$ of $\mathrm{DQB1}{ }^{*} 0201$ negative individuals had detectable levels of IgG4 above the cut-off compared to only $11.1 \%(1 / 9)$ of DQB1*0201 positive individuals, although this difference was not significant $(P<0.05)$. No differences in IgG4 levels were observed between individuals with an active infection (egg-positive). There were no significant differences in the levels of the other antibody isotypes evaluated, either in egg-positive or egg-negative subjects nor with regard to their DQB1*0201 status.

High IgG4 serum levels have been related to advanced stage liver fibrosis in S. mansoni infections (Bonnard et al., 2004; Silveira et al., 2002). In addition, Hagan and colleagues reported epidemiological evidence that low serum IgE and high serum IgG4
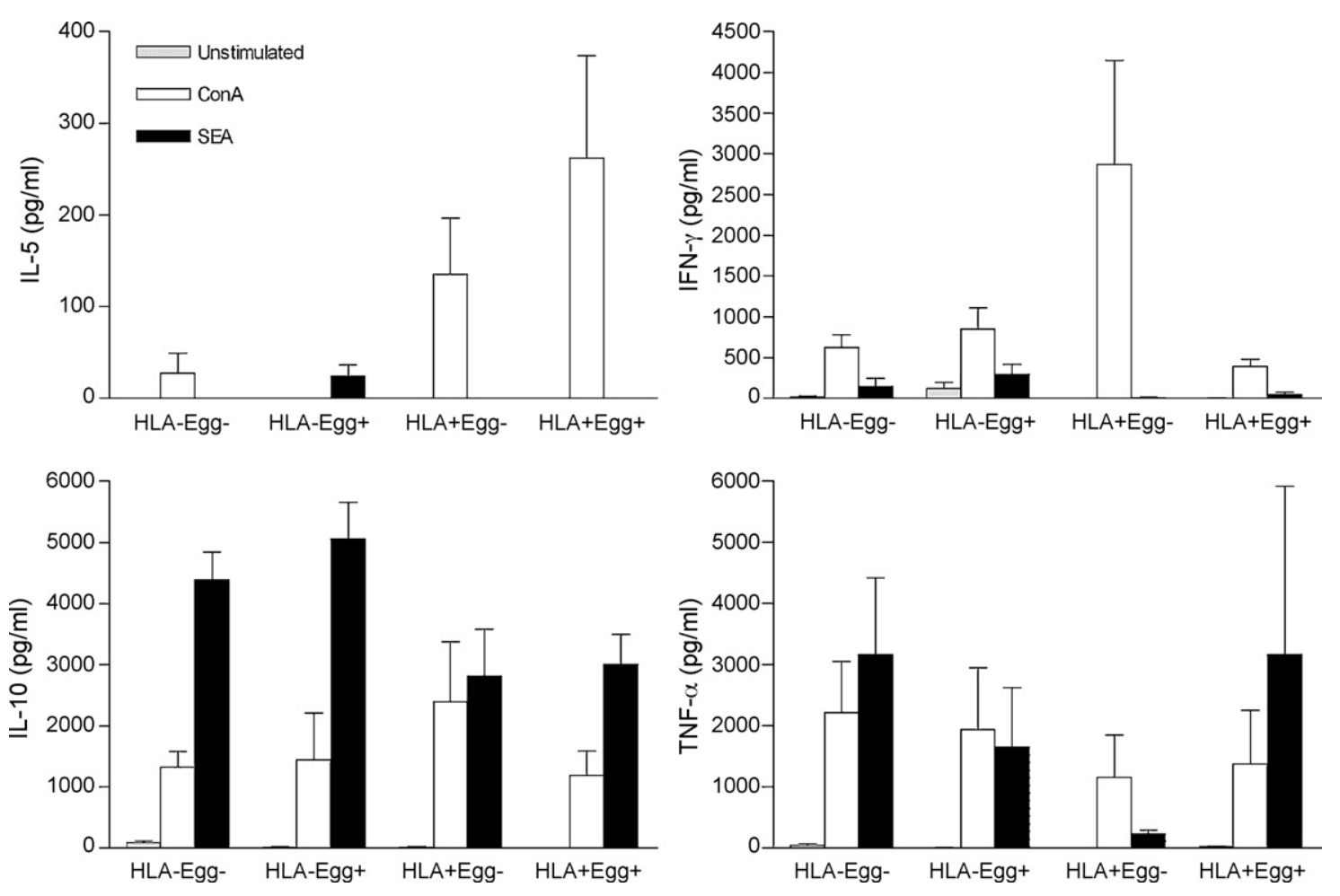

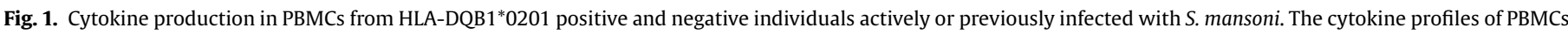

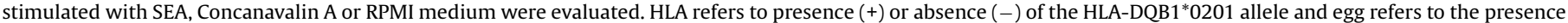
$(+$, active infection) or absence (-, previous infection) of eggs in stool samples of individuals in the study population. The vertical bars represent the mean \pm SEM. 


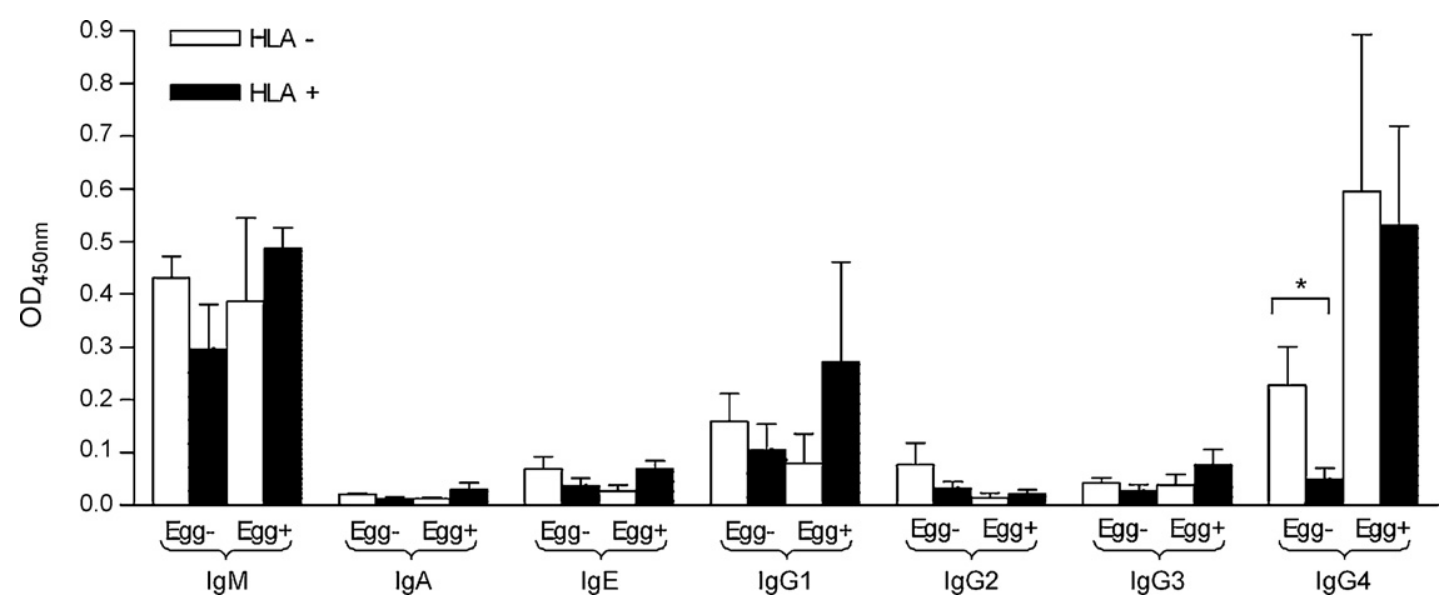

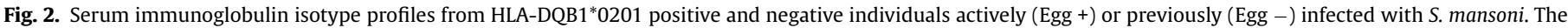
vertical bars represent the mean \pm SEM and the * indicates a statistically significant difference $(P<0.05)$ between the compared groups.

levels correlated with re-infection by Schistosoma haematobium ( $S$. haematobium) (Hagan et al., 1991). Subsequently, similar observations were reported for S. mansoni (Demeure et al., 1993; Caldas et al., 2000). This is likely a consequence of early excessive production of IgG4 antagonizing the potentially protective IgE immunological pathways (Hagan et al., 1991). The influence of HLA polymorphisms on antibody isotypes in schistosomiasis was recently reported. The DRB1*13 allele was associated with higher, post-treatment, levels of IgE against various schistosoma antigens and with a lower frequency of re-infection (Booth et al., 2006). The majority of studies on IgG4 levels have been hampered by the high frequency of undetectable levels of this isotype in young individuals, especially children (Herrod, 1993; Moss et al., 1992). In the present report the mean age of the egg-negative group was $35 \pm 14$ and $32 \pm 08$ for DQB $1 * 0201$ positive and negative individuals, respectively.

In the present work we could not find a plausible relationship between the HLA-DQB1*0201 allele and its effect on the immune response and, more specifically, on cytokine profiles. Low levels of IgG4 among previously infected $\mathrm{DQB} 1{ }^{*} 0201$ carriers suggest that individuals with a previous history of infection are less susceptible to re-infection and this may be an important factor in the development of hepatosplenic disease (Demeure et al., 1993; Caldas et al., 2000). Since the presence of HLA-DQB1 ${ }^{*} 0201$ is associated with hepatosplenic disease (Secor et al., 1996) and high serum IgG4 is indicative of progression to liver fibrosis (Bonnard et al., 2004; Silveira et al., 2002), the results of our study imply that the susceptibility of DQB $1 * 0201$ positive individuals to re-infection is independent of IgG4 levels.

In the present study, we did not detect any significant differences in the immune response during active infection and DQB $1{ }^{*} 0201$ status, however, our study population may not have been large enough to detect such an association due to the small number $(n=10)$ of egg-positive (re-infected) patients. Further studies involving larger numbers of actively infected individuals will be required to clarify the interactions between host genetic background, immune response and susceptibility to severe forms of disease.

\section{Acknowledgments}

This work was supported by grants AI-30639 and AI-0546484 from the National Institutes of Health NIH-USA, and from FINEP/PRONEX 4196086200. We thank Claudio Roberto Santos for technical support at the Itaquara field site.

\section{References}

Alves Oliveira, L.F., Moreno, E.C., Gazzinelli, G., Martins-Filho, O.A., Silveira, A.M., Gazzinelli, A., Malaquias, L.C., LoVerde, P., Leite, P.M., Correa-Oliveira, R., 2006. Cytokine production associated with periportal fibrosis during chronic schistosomiasis mansoni in humans. Infect. Immun. 74, 1215-1221.

Andrade, Z.A., 2004. Schistosomal hepatopathy. Mem. Inst. Oswaldo Cruz 99, 51-57.

Assaad-Khalil, S.H., Helmy, M.A., Zaki, A., Mikhail, M.M., el-Hai, M.A., el-Sawy, M., 1993. Some genetic, clinical and immunologic interrelations in schistosomiasis mansoni. Ann. Biol. Clin. (Paris) 51, 619-626.

Blanton, R.E., Salam, E.A., Ehsan, A., King, C.H., Goddard, K.A., 2005. Schistosomal hepatic fibrosis and the interferon gamma receptor: a linkage analysis using single-nucleotide polymorphic markers. Eur. J. Hum. Genet. 13, 660-668.

Bonnard, P., Remoue, F., Schacht, A.M., Deuffic-Burban, S., Dompnier, J.P., Elguero, E., Charrier, N., Cassagnou, M., Diop, M., Ly, A., Capron, A., Riveau, G., 2004. Specific isotype immune response in the diagnosis of human schistosomiasis pathology? Am. J. Trop. Med. Hyg. 71, 202-205.

Booth, M., Shaw, M.A., Carpenter, D., Joseph, S., Kabatereine, N.B., Kariuki, H.C., Mwatha, J.K., Jones, F.M., Vennervald, B.J., Ouma, J.H., Dunne, D.W., 2006. Carriage of DRB1*13 is associated with increased posttreatment IgE levels against Schistosoma mansoni antigens and lower long-term reinfection levels. J. Immunol. 176, 7112-7118.

Cabello, P.H., Krieger, H., Lopes, J.D., Sant'Ana, E.J., 1991. On the association between HLA-A1 and B5 and clinical forms of schistosomiasis mansoni. Mem. Inst. Oswaldo Cruz 86, 37-40.

Caldas, I.R., Correa-Oliveira, R., Colosimo, E., Carvalho, O.S., Massara, C.L., Colley, D.G., Gazzinelli, G., 2000. Susceptibility and resistance to Schistosoma mansoni reinfection: parallel cellular and isotypic immunologic assessment. Am. J. Trop. Med. Hyg. 62, 57-64.

de Jesus, A.R., Magalhaes, A., Miranda, D.G., Miranda, R.G., Araujo, M.I., de Jesus, A.A., Silva, A., Santana, L.B., Pearce, E., Carvalho, E.M., 2004. Association of type 2 cytokines with hepatic fibrosis in human Schistosoma mansoni infection. Infect. Immun. 72, 3391-3397.

Demeure, C.E., Rihet, P., Abel, L., Ouattara, M., Bourgois, A., Dessein, A.J., 1993. Resistance to Schistosoma mansoni in humans: influence of the IgE/IgG4 balance and IgG2 in immunity to reinfection after chemotherapy. J. Infect. Dis. 168, 1000-1008.

Farah, I.O., Mola, P.W., Kariuki, T.M., Nyindo, M., Blanton, R.E., King, C.L., 2000. Repeated exposure induces periportal fibrosis in Schistosoma mansoni-infected baboons: role of TGF-beta and IL-4. J. Immunol. 164, 5337-5343.

Hafez, M., Aboul Hassan, S., el-Tahan, H., el-Shennawy, F., Khashaba, M., al-Tonbary, Y., el-Morsi, Z., el-Sallab, S., el-Desoky, I., el-Shazly, A., et al., 1991. Immunogenetic susceptibility for post-schistosomal hepatic fibrosis. Am. J. Trop. Med. Hyg. 44, 424-433.

Hagan, P., Blumenthal, U.J., Dunn, D., Simpson, A.J., Wilkins, H.A., 1991. Human IgE, IgG4 and resistance to reinfection with Schistosoma haematobium. Nature 349, 243-245.

Harn, D.A., Danko, K., Quinn, J.J., Stadecker, M.J., 1989. Schistosoma mansoni: the host immune response to egg antigens. I. Partial characterization of cellular and humoral responses to pI fractions of soluble egg antigens. J. Immunol. 142, 2061-2066.

Herrod, H.G., 1993. Management of the patient with IgG subclass deficiency and/or selective antibody deficiency. Ann. Allergy 70, 3-8.

Hirayama, K., Chen, H., Kikuchi, M., Yin, T., Gu, X., Liu, J., Zhang, S., Yuan, H., 1999. HLA-DR-DQ alleles and HLA-DP alleles are independently associated with susceptibility to different stages of post-schistosomal hepatic fibrosis in the Chinese population. Tissue Antigens 53, 269-274. 
Li, Z., King, C.L., Ogundipe, J.O., Licate, L.S., Blanton, R.E., 1995. Preferential recognition by human IgE and IgG4 of a species-specific Schistosoma haematobium serine protease inhibitor. J. Infect. Dis. 171, 416-422.

Montenegro, S.M., Miranda, P., Mahanty, S., Abath, F.G., Teixeira, K.M., Coutinho, E.M. Brinkman, J., Goncalves, I., Domingues, L.A., Domingues, A.L., Sher, A., Wynn, T.A., 1999. Cytokine production in acute versus chronic human Schistosomiasis mansoni: the cross-regulatory role of interferon-gamma and interleukin-10 in the responses of peripheral blood mononuclear cells and splenocytes to parasite antigens. J. Infect. Dis. 179, 1502-1514.

Moss, R.B., Carmack, M.A., Esrig, S., 1992. Deficiency of IgG4 in children: association of isolated IgG4 deficiency with recurrent respiratory tract infection. J. Pediatr. 120, 16-21.

Mwatha, J.K., Kimani, G., Kamau, T., Mbugua, G.G., Ouma, J.H., Mumo, J., Fulford, A.J., Jones, F.M., Butterworth, A.E., Roberts, M.B., Dunne, D.W., 1998. High levels of TNF, soluble TNF receptors, soluble ICAM-1, and IFN-gamma, but low levels of IL5 , are associated with hepatosplenic disease in human schistosomiasis mansoni. J. Immunol. 160, 1992-1999.

Pearce, E.J., MacDonald, A.S., 2002. The immunobiology of schistosomiasis. Nat. Rev. Immunol. 2, 499-511.
Reiman, R.M., Thompson, R.W., Feng, C.G., Hari, D., Knight, R., Cheever, A.W. Rosenberg, H.F., Wynn, T.A., 2006. Interleukin-5 (IL-5) augments the progression of liver fibrosis by regulating IL-13 activity. Infect. Immun. 74 1471-1479.

Salam, E.A., Ishaac, S., Mahmoud, A.A., 1979. Histocompatibilty-linked susceptibility for hepatospleenomegaly in human schistosomiasis mansoni. J. Immunol. 123 , 1829-1831.

Secor, W.E., del Corral, H., dos Reis, M.G., Ramos, E.A., Zimon, A.E., Matos, E.P., Reis, E.A., do Carmo, T.M., Hirayama, K., David, R.A., David, J.R., Harn Jr., D.A., 1996 Association of hepatosplenic schistosomiasis with HLA-DQB1*0201. J. Infect. Dis. 174, 1131-1135.

Silveira, A.M., Bethony, J., Gazzinelli, A., Kloos, H., Fraga, L.A., Alvares, M.C., Prata, A Guerra, H.L., Loverde, P.T., Correa-Oliveira, R., Gazzinelli, G., 2002. High levels of IgG4 to Schistosoma mansoni egg antigens in individuals with periportal fibrosis. Am. J. Trop. Med. Hyg. 66, 542-549.

Velupillai, P., dos Reis, E.A., dos Reis, M.G., Harn, D.A., 2000. Lewis(x)-containing oligosaccharide attenuates schistosome egg antigen-induced immune depression in human schistosomiasis. Hum. Immunol. 61, 225-232. 